Patrice Melé, « ¿Qué producen los conflictos urbanos? », en, F. Carrión, J. Erazo (coord.) , 2016. EL derecho a la ciudad en América Latina, Visiones desde la política. México: UNAM, Coordinación de Humanidades, PUEC, CIALC, IDRC/CRDI, p.127-158. Versión de autor.

\title{
¿Qué producen los conflictos urbanos?
}

\author{
Patrice Melé ${ }^{1}$
}

Este texto propone una reflexión sobre los efectos de la multiplicación y de la generalización de situaciones de conflicto en las ciudades contemporáneas $^{2}$. En América Latina, como en otros contextos, es muy difícil reconstituir la evolución de la conflictualidad; los estudios disponibles son en su mayoría estudios cualitativos y las tentativas de contabilizar o de mapear a escala de las metrópolis, los observatorios de conflictos, son a la vez muy interesantes y decepcionantes ${ }^{3}$. Registran y localizan eventos conflictivos pero ponen en el mismo plano situaciones muy diferentes y no dicen nada ni de la amplitud, ni de la temporalidad de los conflictos. Resulta complicado reconstituir tendencias generales de las dinámicas de los conflictos, lo que se puede decir es que presenciamos una generalización de situaciones conflictivas. Estas, a pesar de presentar muchas diferencias, muestran grupos movilizados que intentan pesar sobre las formas de las dinámicas urbanos o las modalidades de la gestión urbana.

\footnotetext{
${ }^{1}$ UMR CITERES, CNRS, Universidad de Tours. patrice.mele@univ-tours.fr

${ }^{2}$ Agradezco a Claudia Cirelli su lectura y comentarios.

${ }^{3}$ Obversatorio de conflicto de la ciudad de Québec, pero también en América Latina Santiago de Chili, Rio de Janeiro, Belo Horizonte, Maceio.
} 
Es conveniente partir de la idea que existe una gran diversidad de situaciones que podemos calificar como conflictos pero también hay una pluralidad de interpretaciones acerca de estas situaciones en el mundo de la ciencias sociales, o en el mundo de los actores de la ciudad y de los profesionales de la gestión urbana o del urbanismo. Existen controversias sobre lo que revelan estas situaciones, sus causas, y las modalidades y debates sobre las formas de contenerlas desde la acción pública.

Sin embargo, estos momentos de oposición parecen escapar a los conceptos o a las categorías construidas para analizarlos. En efecto, los objetivos de los grupos movilizados son múltiples: permanecer en un lugar, obtener servicios urbanos, luchar contra actividades que producen molestias o riesgos, imposibilitar la realización de un proyecto, controlar el tipo de urbanización, guardar el uso exclusivo de espacios públicos y de ciertos recursos o proteger ciertos espacios.

Los conflictos urbanos pueden ser calificados de luchas por la tierra y los servicios urbanos, de movilizaciones ambientales o patrimoniales, de oposición a proyectos públicos o a la autorización de ciertos usos urbanos, de antagonismos entre grupos por el uso del suelo, o de movimientos políticos enfocados a los efectos locales de tendencias globales. Se tiene que subrayar también que observamos en los espacios públicos de las ciudades la presencia de movimientos sociales, que no tienen la ciudad como objetivos, bajo la forma de marchas o plantón. La investigación de situaciones concretas muestra una imbricación de las causas; muchas veces se juntan vecinos movilizados que constituyen redes con grupos o militantes que reivindican movimientos sociales en nombre de la justicia espacial, del reconocimiento de la cualidad ambiental de un espacio, del acceso al espacio público como recursos o de la preservación de su calidad de vida.

Entonces, la investigación urbana debe enfrentarse al reto de saber si tiene sentido agrupar analíticamente en una sola categoría conflictos urbanos tan diferentes. La posición que desarrollaré aquí es la de plantear que, para analizar la relación entre dinámicas urbanas y sociales, es de gran interés construir hipótesis de interpretación sobre los efectos de estas situaciones de conflicto y no solamente sobre sus causas o lo que revelan de tendencias estructurales de las sociedades contemporáneas.

He intitulado este texto ¿Qué producen los conflictos urbanos? porque lo que quisiera proponer es presentar mi posición sobre la cuestión esta cuestión a partir de investigaciones personales (Melé 2014), colectivas en México (Bassols, Melé 2001), en Europa o en procesos comparativos internacionales (Bourdin, Lefeuvre, Melé 2006 ; Melé 2012 ; 2013) ${ }^{4}$. Estos

${ }^{4}$ En estas investigaciones colectivas participaron varios colegas, quisiera recordar aquí antes que nadie a Emilio Duhau con el cual he intercambiando durante muchos años puntos de vista sobre el tema de la conflictualdad urbana y que ha escrito textos muy estimulantes sobre este tema (Duhau 2012 ; Duhau, Giglia 2008). Al análisis de las situaciones mexicanas participaron Antonio Azuela, Jade Latargère, 
proyectos plantearon estudiar situaciones de conflicto bajo el punto de vista de los efectos y discutir hipótesis de interpretación sobre lo que producen las situaciones de conflictos. En efecto, se trata de subrayar la importancia de reflexionar sobre lo que revelan estos hechos de las relaciones entre los actores, la forma de contenerlos o de dar más espacios en las movilizaciones. Este texto está organizado en dos partes. En primer lugar, presentaré algunas posiciones, prerrequisitos que considero necesario para construir una reflexión sobre la productividad de las situaciones de conflictos, en segundo lugar, algunas hipótesis de interpretación sobre la productividad territorial, jurídica y política de los conflictos urbanos a manera de síntesis de los resultados de estas investigaciones.

\section{Posiciones para el estudio de los conflictos urbanos}

La construcción de una inteligibilidad de las situaciones de conflicto tiene que cuidarse del impacto de dos formas opuestas de interpretación que, para mí, no permiten aprehender los efectos de la multiplicación de los conflictos. En primer lugar, hay que cuidarse de una sobrecarga teórica, es decir del papel de las explicaciones macro teóricas que presuponen causas estructurales a los conflictos o que insertan el conflicto en teorías del cambio social. No es que estas teorías no tengan elementos de validez sino porque me parece que tienen consecuencias sobre las investigaciones concretas que conllevan a descalificar las movilizaciones que no corresponden a los criterios normativos que establece. La literatura científica sobre los conflictos urbanos está marcada por el papel que la sociología de inspiración marxista otorga a lo que ha definido como nuevos movimientos sociales urbanos (Castells, 1974). O más recientemente por el peso de una geografía critica marxista de tradición anglófona (Harvey 2013, Soja 2010) que conceptualiza los conflictos urbanos a partir de su capacidad para enfrentar los impactos de la hegemonía neo-liberal sobre las ciudades. Estos enfoques tienen bastante influencia actualmente en Francia y en América Latina, y paradójicamente tienen el efecto de ignorar los importantes debates sobre estos temas y los aportes de la escuela de sociología urbana marxista de lengua francesa o español que tuvo un gran peso a finales de los 1970 y 1980 (Amiot 1986).

En estos trabajos, los movimientos sociales urbanos representan nuevas formas de lucha en defensa de un marco de vida de calidad, y son dotados de un papel activo y determinante para el cambio social. Uno de los efectos de esta postura ha sido de poner las movilizaciones urbanas en el centro de

Angela Giglia, Vicente Ugalde y, entre otros, para los casos en Francia, Claudia Cirelli, Laurence Rocher ; y entre otros en Montréal Annick Germain y en Italia Luigi Bobbio, Egedio Dansero y Fabrizio Maccaglia. Estos proyecto fueron financiados por el Agencia nacional de la investigación francesa, el programa Concertación, decisión, ambiente, del ministerio del ambiente francés y del ADEME, además una parte de los viajes Mexico Francia fueron financiados gracias al programa de intercambio con el Colegio de Mexico (ECOS NORD / ANUEIS / CONACYT). 
una análisis de las contradicciones urbanos, de las relaciones entre el Estado y las clases sociales (Valladares, Prates Cuelho 1995, 13). Sin embargo, este modelo de interpretación fue muy criticado desde los años 1980 , por una parte, en lo que concierne el concepto de Estado considerado como una entidad y no como un campo de lucha de diferentes grupos, $y$, por otra parte, por la cuestión de la reproducibilidad de un modelo de análisis que fue construido a partir de la identificación del papel de las políticas urbanas en el "capitalismo monopolístico de estado" (Valladares, Prates Cuelho 1995). Los investigadores que se inscriben en este marco de análisis buscan vincular las movilizaciones locales a grandes tendencias del cambio social y político. Este tipo de marco teórico ha producido una visión unificada de las luchas urbanas que se ha denominada "movimiento urbano popular" (utilizando el singular) (Moctezuma 1994). Esta posición teórica ha permitido que trabajos sobre conflictos y movilizaciones urbanas sean integrados en enfoques globales de los movimientos sociales con el objetivo de analizar la posibilidad de una convergencia de las diferentes luchas. Este marco ha sido posteriormente aplicado y llevado al campo, por militantes o investigadores comprometidos con las movilizaciones que encuentran de esta manera al mismo tiempo un sentido histórica tanto en los movimientos como en su compromiso.

Sin discutir aquí la validez y los aportes de estas posición y de la inserción de las acciones colectivas urbanas en las macro-teorías del cambio social, se tiene que subrayar que estos enfoques, como los trabajos de Alain Touraine (Touraine 1973), proponen definiciones normativas, más o menos abiertas, de las cualidades y de las formas que tienen que tener una movilización para volverse un verdadero movimiento social o un movimiento social urbano, o un movimiento realmente anti-capitalista. Lo anterior produce la deslegitimación de otras formas de acción colectivas que no corresponden al modelo o que "todavía non han alcanzado" características que permitieran transfórmalas en verdadero movimiento social.

A partir de 1983, Manuel Castells (1983), en una obra crítica de sus propias posiciones y del marxismo urbano, propuso una conceptualización diferente de los movimientos urbanos en la construcción de un marco de análisis comparativo de las movilizaciones urbanas. El objetivo del autor ya no es la construcción de una teoría general del cambio social, sino limitar el análisis a las relaciones entre movilizaciones urbanas y cambio urbano. La situación actual y la reaparición de las teorías marxistas en los estudios urbanos bajo la influencia de la geografía radical plantea una paradoja. Por una parte, genera un nuevo interés por el estudio de los conflictos urbanos pero, al mismo tiempo, limita el análisis de las dimensiones políticas de estas situaciones, ya que se enfoca en las dimensiones económicas y en los vínculos entre conflictualidad y recomposición neoliberal del capitalismo. 
Obviamente hay también otras macro-teorías que tienen un impacto sobre los estudios del conflicto :

1 - Podemos subrayar el papel de las teorías sociológica de la dominación, que se refieren explícitamente a la sociología de Pierre Bourdieu, y que buscan el origen estructural de los conflictos en las relaciones de dominación (Moya 1982). Lo anterior lleva también a insistir sobre las relaciones asimétricas y desigualdades al interior de los movimientos y a veces, a demostrar que los movilizados no son los más dominados o los más pobres. Este marco teórico conlleva una cierta forma de deslegitimación de los actores movilizados por falta de autonomía o por reproducir relación de dominación al interior de los conflictos. La observación de situaciones de conflictos se vuelve un campo más de la identificación de la existencia de principios estructurales que la acción colectiva no puede rebasar (Messu 2012).

2 - De forma distinta encontramos también el peso de la nueva sociología de los movimientos sociales que se ancla en la ciencia política y la historia contemporánea (Goirand 2010): la corriente llamada política del conflicto en referencia al trabajo de Charles Tilly y Sidney Tarrow (2006), propone un marco de análisis interesantes pero muy formalizado, permite comparar conflicto urbano o ambiéntales con otros tipos de movimientos y hacer comparación transnacionales y trans-histórica a partir de un mismo vocabulario y de una serie de conceptos (estructura de las oportunidades políticas, organización y recursos, repertorio de acción, análisis de marco). Este influente grupo de investigación que a veces es criticado por su rigidez y su voluntad de estructurarse como una disciplina (Fillieule, Mathieu, Pechu 2009) actualmente parece tener menos influencia en América Latina que en Europa y sobre la investigación de países anglófonos.

Para construir una mirada sobre los efectos de las situaciones de conflicto que abarque la gran diversidad de experiencias se tiene que postergar la interpretación en lo que concierne les efectos macro-sociales o macropolíticos. Lo que no significa rechazar la posibilidad de proponer hipótesis de interpretación sobre las relaciones entre conflicto, política y derecho a la ciudad. Lo que intentare hacer en la parte final de este texto.

Contrariamente a esta sobrecarga teórica de los conflictos urbanos existe otra forma de conceptualización que a la vez denomina y descalifica las movilizaciones que tenemos que dejar a un lado. Se trata de las precalificaciones de estas situaciones como manifestaciones de un síndrome NIMBY ${ }^{5}$ (Dear 1992 ; Wolsink 1994). En efecto, los conflictos de

\footnotetext{
${ }^{5}$ NIMBY: Not in my backyard, no en mi patio tracero, categoría introducida por planificadores de Estados Unidos para descalificar los conflictos de residentes que rehúsen la localización cerca de su lugar de residencia de ciertas actividades o de ciertas infraestructuras.
} 
proximidad son muchas veces interpretados como manifestaciones de una tendencia generalizada al repliegue de los habitantes sobre su espacio de residencia lo que podría contribuir a la fragmentación urbana. El problema de este tipo de interpretación, no es de saber si las movilizaciones son realmente egoístas o sordas al interés general, es que propone una conclusión al mismo tiempo que identifica un fenómeno. Se trata de una apelación que deslegitima los vínculos que resultan de la proximidad y las posiciones de los grupos movilizados. El éxito del acrónimo, que se basa en su apariencia de diagnóstico y revela a partir de los años 1980, la difusión de una tendencia social ya bien establecida en Estados Unidos, ha ocultado la complejidad de esos procesos de acción colectiva, los cuales ponen en juego no solamente las relaciones entre los habitantes y el espacio cercano a su vivienda sino también sus relaciones con los objetivos y procedimientos de las acciones de los poderes públicos.

La conceptualización de NIMBY está ligada a otro concepto, el de la generalización ${ }^{6}$. Se trata de uno de los marcos de análisis de las modalidades de acceso al espacio público de las movilizaciones de vecinos. En este marco se presupone que los habitantes tienen que realizar una generalización de sus argumentos para anclar su movilización en valores patrimonio, medio-ambiente, justicia - que permite escapar a esta descalificación y acceder al espacio público de debate (Trom 1999). La generalización de esta forma de pensar los conflictos implica una visión por la cual el interés particular del propietario, del habitante, del usuario funda la movilización. Es, en un segundo tiempo, cuando los habitantes son confrontado a pruebas ( contactos con la prensa, con funcionarios públicos, con avocados o expertos); después de estos procesos adquieren la capacidad de argumentar ya que son son sometidos a imperativos de justificación (Thévenot 2006) que les obliga a anclar su movilización en valores.

Es posible construir una crítica de esta visión. En efecto, en muchas ocasiones nuestras encuestas mostraron que el anclaje en valores está presente desde el principio de la movilización, en particular desde la constitución de los grupos movilizados. Varios estudios y en particular los que he podido llevar a cabo en México (Melé 2014), muestran que las movilizaciones pueden ser analizadas como construcción de redes híbridas lo que implica la movilización de vecinos, actores políticos y institucionales, militantes políticos o ecológicos, que están presente desde un principio. Entonces, más que pensar las cosas como un procesos estratégico de generalización ; se tendría que pensar la difusión, en la hibridación de las posiciones de los diferentes actores en el marco de las movilización y la construcción de diferentes formas de alianzas.

Quisiera plantear aquí la posibilidad de apartarse de estas explicaciones previas o marco de análisis de los conflictos, para dejar abiertas las interpretaciones. En investigaciones, basadas en estudios de casos, hemos

\footnotetext{
${ }^{6}$ En francés : "montée en généralité"
} 
decidido partir de un imperativo de descripción fina acerca de las situaciones, de los actores movilizados, de sus contextos para intentar estudiar los efectos de los conflictos: es una invitación a una reflexión sobre la productividad social de los conflictos. No hay espacios en este capítulo para hacer referencias a los casos estudiados, el lector interesado podrá referirse a los libros publicado a partir de los procesos de investigación citados.

\section{Analizar los efectos de los conflictos urbanos}

La posición teórica sobre los conflictos que defiendo aquí considera que el conflicto no se debe aprehender únicamente como una crisis sino que, como lo demostró Georg Simmel (2010 [1903]), es consustancial al funcionamiento de las sociedades; constituye una forma positiva de socialización. Multiplicando las interacciones entre participantes, los conflictos producen asociaciones, alianzas, coaliciones y redes. Esta forma social puede entonces ser considerada como un fenómeno que « reduce el aislamiento social " que " agrupa parte " para retomar el vocabulario de Lewis Coser (1982 [1956]). Los trabajos de los sociólogos que suscriben a esta posición subrayan el papel de estructuración social de los conflictos.

Desde esa óptica, hoy en día se estudia el nuevo lugar que ocupan los conflictos tanto en las relaciones entre, por una parte, los residentes (o usuarios) y la acción pública, como en las relaciones entre los habitantes y el espacio próximo. Mientras los conflictos sociales y políticos han sido estudiados tradicionalmente desde el punto de vista de la acción colectiva, las formas de movilización, las ideologías y los valores que defienden y de la organización o la racionalidad de los actores movilizados, me parece que la comprensión de ese tipo de situación debe pasar por un examen pragmático de lo que producen. Esto significa reflexionar sobre sus efectos en la recomposición de las modalidades de territorialización de la acción pública y de la localización de las diferentes infraestructuras, sobre su función en la apropiación del espacio próximo por los residentes, pero también de la relación de los grupos movilizados con lo jurídico y lo político. Este texto hace énfasis en tres dimensiones de la productividad de los conflictos : la productividad territorial, la productividad jurídica y la productividad política.

\section{La productividad territorial de los conflictos urbanos}

La reflexión geográfica se interesa a las movilizaciones locales a partir de sus capacidades a revelar 0 a fortalecer territorialidades pre-existentes (Melé, Larrue, Rosemberg 2003). Las movilizaciones, controversias y conflictos son estudiados come reveladores de anclajes territoriales que reenvían a la pertenencia, la apropiación o inclusive a la identidad territorial. Sobre estas cuestiones, he intentado adoptar una postura diferente y focalizar la atención sobre el análisis de los efectos de las movilizaciones locales, sobre su productividad espacial o territorial. 
En efecto, podemos decir que las movilizaciones locales constituyen non solamente una forma de territorialización puesto que producen grupos conscientes de una solidaridad de destino que replantean su inscripción espacial, que se apropian materialmente de un espacio gracias a un trabajo de vigilancia localizada y idealmente por un trabajo de nominación y de valorización.

Esta vigilancia puede ser analizada, en el sentido de la etología, como una conducta territorial de toma de posición de un espacio o de su defensa contra otros usuarios (Le Berre, 1992), o como una manifestación de " territorialidad » según la tradición de la escuela de Chicago (Stanford, Scott, 1967); en esta concepción, la territorialidad es una tentativa de control sobre el espacio (Sack, 1986). Además, las redes de habitantes que desarrollan una acción colectiva practican una sociabilidad con un fuerte anclaje espacial, constitutivo de otra forma de territorialidad.

Por otra parte, para defender su posición, los habitantes movilizados identifican cualidades o valores del espacio en juego y efectúan un trabajo de producción de una cierta representación de su espacio residencial. Esta producción / promoción del territorio refuerza la territorialidad específica producida por la acción colectiva. Se podría decir también que las movilizaciones locales construyen simbólicamente el espacio próximo como un bien común, que crea entre un grupo así constituido y objetos, lugares o conjuntos espaciales una relación patrimonial (Barel, 1984), lo que en otro vocabulario se podría llamar apropiación.

Observamos también la multiplicación de instancias localizadas que se plantean como objetivo construir la aceptación o (re)construir la confianza entre los diferentes actores o portadores de intereses (mesas de negociación, comisión local de seguimiento de cierta actividad, etc...). La existencia de estas formas de regulaciones locales, implementadas a veces sobre perímetros muy parecidos a los de las movilizaciones, nos obligan a considerar que asistimos a una producción de territorios en el sentido de espacios delimitados cargados de valores y dotados en ciertos casos de una instancia - de gestión y de concertación - en las cuales los habitantes movilizados son representados. Estos que se presentan como voceros del espacio en juego; pero también como actores de la actualización local de las reglas jurídicas. Se apropian textos de leyes y objetivos de las políticas públicas que utilizan como recursos de la situación local.

Los grupos movilizados construyen un discurso sobre los límites de las calificaciones jurídicas del espacio (zonificaciones) y elaboran reivindicaciones implicando la implementación de disposiciones jurídicas territorializadas. En este sentido, podemos decir que los habitantes movilizados contribuyen a la transmisión de una visión territorial del espacio por integración / difusión de los procedimientos y de las categorías de la acción pública. Entonces, no es solamente la acción colectiva sobre une base espacial que constituye un proceso de territorializacion. Pero si, la doble experiencia de la construcción de un actor colectivo en el momento de la movilización y de su confrontación a los procedimientos territorializados 
de la acción pública. La integración del vocabulario, de las referencias y de las tipificaciones portadas por el derecho y la acción pública implica la adopción de una visión territorial del mundo.

\section{Un régimen de territorialidad reflexiva}

En las movilizaciones locales, grupos y redes constituidos en partes de residentes, identifican por un trabajo de encuesta, elementos valorizables de su espacio que pueden justificar su protección. Realizando este proceso ellos adquieren y comparten une cierta visión de su espacio próximo. Podemos plantear la hipótesis que esta producción de conocimiento y esta puesta en debate del futuro (devenir) de un conjunto espacial tiene efectos a la vez sobre las modalidades de la acción de los poderes públicos y sobre las relaciones al espacio de las habitantes movilizados.

Más que de territorialización reactiva (Lolive, 1999) estos procesos pueden ser calificados de territorialización reflexiva (Melé 2009) lo que significa que estos grupos construyen una escena en la cual son debatidas las informaciones sobre las calidades, las evoluciones y el porvenir posible de un territorio y proclaman la necesidad de la implementación de procedimientos de participación y de seguimiento - de reflexividad institucional (Giddens, 1995, 2008) - para intentar reducir las incertidumbres ligadas a las dinámicas urbanas y a los usos de suelo.

Se puede conceptualizar la reflexividad como la capacidad de los actores a entender lo que hacen cuando lo hacen y a dar cuenta y analizar sus actividades. Esta conciencia práctica autoriza la adaptación al contexto y al curso de la acción. A otro nivel, hace posible la producción de las descripciones de los objetivos y de las razones de la acción y funda su capacidad de adaptación al contexto. Podemos retomar aquí la definición de Anthony Giddens (1995) que considera que la reflexividad de la vida social moderna, consiste en una revisión constante de las prácticas sociales a la luz de las informaciones nuevas que conciernen estas prácticas, lo que altera de esta forma su carácter.

Podemos considerar que esta presunción de reflexividad - es decir la conciencia del carácter limitado de todo saber - disuelve la asimilación entre saber y certidumbre. Se puede observar que procesos de reflexividad institucional que mejoran el nivel de conocimiento se multiplican (sistemas de evaluación del riesgo, dispositivos de seguimiento en tiempo real, comisión de coordinación, de información o de seguimiento, diferentes formas de urbanismo participativo), sin que estas puedan construir un universo de certidumbre, más bien conducen a la toma de conciencia del carácter inestable del mundo social. Esto lo subraya también Ulrich Beck (2006) en su análisis de las relaciones entre saber científicos y riesgos

Es posible identificar procesos similares ligados a la fuerte presencia en los conflictos urbanos del medio ambiente a la vez como valores y marco de acción. El medio-ambiente conduce a redefinir bienes comunes y los objetivos y modalidades de la acción de los poderes públicos para preservarlos, ya que esta noción se basa en la identificación de los lazos y 
retro-acción entre un individuo y lo que lo rodea. De una manera diferente, las referencias al patrimonio implican también una relación reflexiva a ciertos objetos, lugares y espacios (Melé 2010 ; Melé 2014).

En este marco de análisis, las situaciones de acción en público que movilizan los valores patrimoniales y ambientales, y en las cuales se debate el porvenir de conjuntos espaciales, pueden ser analizadas como manifestaciones de reflexividad societal. En efectos, los legos se reapropian saber expertos y (re)construyen a partir de estos su relación al espacio y a las acciones de los poderes públicos.

Los habitantes movilizados realizan igualmente una crítica de los objetivos de la intervención pública, que requiere de los actores públicos une capacidad de análisis permanente del curso y de los impactos de su acción, y una toma en cuenta de los efectos no-intencionales de las actuaciones.

Las negociaciones o la generalización de instancias de debates o de concertación no son en capaces de aportar certidumbres para resolver los conflictos, ya que estas estrategias constituyen la institucionalización de escenas publicas que integran primero la vigilancia de los residentes $y$, después, procesos de hibridación de las saberes profanos al contactos de los actores públicos. De cierta forma, para retomar el vocabulario de Giddens estas instancias forman puntos de encuentro entre reflexividad individual y colectiva (investigación de los residentes afectados) y procedimientos de reflexividad institucional.

Podemos construir la hipótesis de la difusión de un régimen especifico de territorialidad que esté caracterizado no por el anclaje, la tradición y el tiempo largo, o por un proyecto de actores institucionales, pero por un lugar particular de la acción, del debate en publicó, de la difusión de modos de conocimiento y de valorización del espacio, así como por la puesta a distancia de sus experiencias cotidianas para transformarla en saber que se puede movilizar en situaciónes publicas.

No se trata aquí de oponer territorialidades antiguas capaces únicamente de inercia, de resistencia, o de resiliencia a territorialidades nuevas, que caracterizan diferentes formas de (re)inversión o de (re)territorializacion, pero de subrayar que, tomando en serio el trabajo de los individuos movilizados, es posible identificar una forma específica de relación al espacio próximo ligado a las acciones colectivas en público en el marco de movilizaciones locales. Podemos, emitir la hipótesis que la multiplicación y la generalización de situaciones de movilización local en las cuales una definición de bienes comunes localizados está en juego constituye una modalidad cada vez más presente de conciencia de sí de ciertos grupos locales. 


\section{La productividad jurídica de los conflictos urbanos ${ }^{7}$}

Una forma de enmarcar el análisis de los conflictos en una reflexión sobre el derecho a la ciudad es centrarse en la relación al derecho y el papel del derecho en estas situaciones de conflicto. En un primer nivel, podemos analizar la relación al derecho de los habitantes o vecinos movilizados en un conflicto ambiental a partir de la experiencia del uso del derecho, de la judicialización de los conflictos. Como lo subraya Antonio Azuela, el contacto con el mundo del derecho y sus representantes es una experiencia compleja, contradictoria, ambivalente (Melé et al. 2013). En este sentido, los conflictos son también un procesos de elaboración colectiva de una situación jurídica, una modalidad de difusión de conocimientos jurídicos. Para anclar su causa en el mundo de los tribunales los grupos movilizados tienen que acudir a especialistas del derecho que realizan un trabajo de traducción de los argumentos que privilegian lo que puede servir de base para una estrategia. Los grupos movilizados hacen la experiencia del peso de los procedimientos, de las estrategias procesuales que tienen a veces más importancia que los argumentos en termino de justicia urbana y ambiental. Debemos subrayar también la experiencia del carácter indeterminado del derecho y de los tiempos largos del camino de la judicialización. En efecto, si muchas veces el derecho se presenta así, se debe subrayar que en pocos casos les conflictos son resueltos por los procedimientos previstos en las leyes. Como los actores hacen también la experiencia de la indeterminación del recurso a los tribunales, no es posible saber cuál puede ser el resultado de una decisión jurídica ni sus efectos. Por otra parte, los tiempos del derecho son largos, ligados a la posibilidad de amparos y recursos a otras instancias. Además el marco jurídico esta, especialmente en el campo ambiental, en evolución rápida. Recurrir a los tribunales permite muchas veces ganar tiempo, frenar una obra, buscar esperar tiempos políticos más propicios; o ya sea un cambio en el marco jurídico más favorable a los oponentes (Azuela, Melé, Ugalde 2015).

Además de estos niveles, es posible considerar, también, la presencia del derecho en las situaciones fuera de todo recurso a los tribunales. Las situaciones de conflicto y las movilizaciones son momentos de exposición de los habitantes movilizados al orden jurídico no solamente cuando recuren a avocados o a tribunales, pero también cuando buscan argumentar frente a la prensa o a actores públicos. Se trata de experiencias que permitan adquirir competencias sobre las diferentes calificaciones jurídicas del espacio y sus efectos reales o simbólicos y se podría decir momentos de socialización jurídica.

\footnotetext{
${ }^{7}$ Cabe subrayar que lo que presentamos aquí se basa en reflexiones comunes desarrolladas conjuntamente con Antonio Azuela y Vicente Ugalde véase (Azuela, Melé, Ugalde, 2015).
} 


\section{Con un texto y su interpretación enfrentarse a los potentes}

Cuando los habitantes apelan al derecho, recurren muchas veces a un documento que garantice sus derechos. Para los habitantes, el derecho existe principalmente como un texto del cual esperan un efecto. Varias situaciones de conflicto en las cuales hemos realizado entrevistas muestran que las personas implicadas en un conflicto no solamente construyen una interpretación del derecho directamente del texto de la ley, sino que experimentaron en situación la fuerza del derecho como texto y su capacidad a movilizarlo.

Referirse y mostrar un documento en un debate o una controversia - sin importar cual sea su validez jurídica - con el propósito de deslegitimar una práctica administrativa o de apoyar una reivindicación constituye una de las experiencias más comunes de esta función del derecho. La experiencia del derecho es, entonces, en este caso la de un cuerpo de textos del cual se puede sacar elementos para fundar (sus) derechos. Por ello, en nuestras entrevistas, el orden jurídico es entonces, en primera instancia, presente bajo la forma de referencias a los textos que se tienen que identificar, entender y interpretar (Melé 2014 ; Melé 2006). Para los habitantes, es importante determinar el texto de referencia: lo que una representante de un grupo de vecinos llama " obtener la información justa ", es poder cualificar jurídicamente una situación por medio de un documento. Cuando han identificado el texto que " se aplica », después de una búsqueda - que puede ser difícil - los habitantes se movilizan para emparejar la realidad con su interpretación jurídica. En sentido pragmático, el texto que se aplica, es el que produce un efecto cuando se lo invoca. Se trata entonces de hacer existir - de actualizar localmente - el marco jurídico, más exactamente los textos de leyes considerados literalmente como afirmaciones explicitas.

Se puede entonces identificar uno de los usos sociales del derecho; una de las características del derecho es, en efecto, ser un texto disponible sometido a interpretación directa por la lectura. Podemos pensar que esta exposición al derecho como texto tiene efectos sobre la racionalidad de los habitantes movilizados, sobre su visión del mundo, sobre la definición de la situación y no solamente de la situación jurídica. Tanto el texto de ley como el reglamento existen en sí mismos de forma independiente a los atributos que lo transforman en políticas públicas (decretos de aplicación, reglamentos internos, procedimientos y prácticas administrativas).

\section{El papel de las calificaciones jurídicas del espacio}

A partir de las investigaciones que se basan sobre situaciones de calificaciones jurídicas del espacio ligados a políticas patrimoniales y ambientales (zonas de monumentos, espacios protegidos, zonas de protección contra riesgos o molestias) podemos decir que el estatuto jurídico creado por estas zonificación es interpretado por los habitantes y vecinos como un reglamento estricto de las evoluciones de un espacio. Calificando ciertos espacios, el derecho crea expectativas sobre las evoluciones de estos espacios y sobre la definición de las prácticas sociales compatibles 
con esta protección. Estas expectativas son determinadas tanto por la representación de los valores movilizados - aquí el patrimonio y el ambiente - o por una cierta concepción de lo que es un entorno de vida aceptable que en las prescripciones normativas precisas ligadas a reglas de derecho territorializadas. La frustración de estas expectativas puede traducirse en controversia o conflicto. Podemos decir que los grupos y los habitantes movilizados se vuelven especialistas de los procedimientos administrativos y de las reglas que establece el derecho de la planeación urbana y de las diferentes tipos de zonificación.

Las personas entrevistadas demuestran, al enseñar mapas extraídos de los diferentes documentos de urbanismo y al dibujar espontáneamente las zonas, un buen conocimiento de los límites y de las zonificaciones. La discusión sobre los límites es un momento álgido de toda negociación, ya que las expectativas sobre el papel de ciertas restricciones territorializadas - consideradas como recursos para luchar contra las molestias - son enormes. El deseo de seguridad, de conservación de la imagen o de las funciones del espacio cercano a la vivienda pasa, a menudo, por una demanda de territorialización de las restricciones jurídicas particulares. Los habitantes movilizados han incorporado una visión territorial de la acción pública en forma de perímetros de acción o de zonificación. Al igual que los interlocutores públicos, utilizan también una definición territorial de la situación que supone, en cuanto a la relación inmediata de cada habitante con el espacio, la capacidad de tomar distancia de su propia situación particular. Por lo tanto, la incorporación de una visión territorial implica por parte de los habitantes una cierta abstracción hacia el plano general, una definición del territorio que presente una posición común en cuanto a las molestias o a los desafíos en materia de patrimonio o ambiente.

En México, en los casos de conflictos ambientales recogidos en la obra Transacciones territoriales (Melé 2014), hemos demostrado que frente a las ventajas proclamadas y a las garantías ambientales aportadas por los promotores de los proyectos, los detractores intentan movilizar recursos y argumentos derivados del orden jurídico y regulatorio. Al intentar definir las reglas de derecho que se pueden activar para la situación local, descubren el mundo de la planificación del territorio, en particular las zonificaciones ambientales y las de ordenación urbana. Estas calificaciones territorializadas hacen valer en derecho una vocación y al mismo tiempo un "deber ser" del espacio. Los detractores intentan entonces reclamar la aplicación estricta de un estatus del espacio definido en los documentos de ordenación.

Generalmente, los residentes movilizados se enteran de la existencia de las zonificaciones que protegen ciertos espacios en el momento del conflicto. La mayoría de las veces son los peritos y/o los miembros de las asociaciones de protección del patrimonio y del ambiente los que desentierran estas calificaciones. Una vez identificada la existencia de una zonificación, el debate se centra sobre sus límites y su validez, así como sobre las razones para el incumplimiento de las reglas que estipula. Prácticamente se evoca siempre la capacidad de los agentes económicos 
para saltarse las reglas de uso del suelo o las protecciones ambientales. Paradójicamente, estas herramientas que territorializan las voluntades de control se han convertido en el símbolo de la incapacidad y/o del abandono de la ambición planificadora.

Una segunda etapa del contacto con estas calificaciones parece ser la comprobación de su capacidad para movilizar a los agentes institucionales responsables. Por ejemplo, en México, la existencia de un parque nacional, incluso cuando se ha creado hace tiempo, obliga a las autoridades federales a desempeñar el papel de garante del futuro de dicho espacio, o, como mínimo, a intentar la justificación del laxismo y a tramitar las quejas por haberse incumplido el destino del parque. En Monterrey, la movilización consiguió impulsar un nuevo proceso de delimitación del parque nacional y la instauración de nuevas reglas de gestión que deberían permitir el establecimiento de límites a la urbanización. Las situaciones analizadas muestran, además, que parece mucho más fácil intentar reactivar calificaciones que ya existen que construir, a partir de la movilización, un nuevo tipo de calificación del espacio. Aunque esto último se consiguió con la declaración del Cerro de la Silla, por parte de la Federación, como "monumento nacional" (Melé 2001).

Incluso cuando no se respetan, estas calificaciones del espacio desempeñan un papel muy importante en la construcción de la legitimidad de las reivindicaciones. De hecho, parecería que para poder oponerse a los promotores de los proyectos y al gobierno con argumentos creíbles referidos a la calificación de un espacio, fuera necesario que dicha calificación se encontrara ya inscrita en el derecho y estuviera reconocida por un mecanismo territorializado. En prácticamente todos los conflictos analizados, la representación del espacio que forjan los habitantes en conflicto queda reforzada por aquellos instrumentos jurídicos y cartográficos que expresan las cualidades del espacio en cuestión.

El contacto de los habitantes con estas calificaciones jurídicas del espacio contribuye a la construcción del valor del subconjunto espacial objeto de dicha distinción. Podemos observar que las zonificaciones presentan la peculiaridad de difundir en la totalidad de un espacio definido territorialmente los valores que las fundamentan. En la práctica, esta capacidad parece más importante que el análisis preciso de los elementos que llevan a optar por la decisión de la zonificación y que las herramientas asociadas.

Aunque las estrategias estéticas o de valorización de la imagen de los lugares pueden suscitar debate y controversia en cuanto a los límites pertinentes o a la compatibilidad con el proyecto, las calificaciones del espacio fundamentadas en el derecho constituyen, sin embargo, los únicos elementos que pueden ser considerados como "no negociables" de la territorialización de los valores del espacio. ${ }^{8}$ Para asentar o dar un contenido

\footnotetext{
${ }^{8}$ En cuanto a la solidez de los límites fundamentados en el derecho, obviamente es necesario matizar esta cuestión recordando que sus delimitaciones, incluso si se basan firmemente en los valores ambientales o en los riesgos, son objeto de
} 
a su búsqueda de valorización del espacio, los residentes intentan aferrarse a las calificaciones jurídicas del espacio que implican la valorización de ciertos espacios y cuya activación podría argumentar una incompatibilidad con el proyecto.

Cuando las colectividades intentan movilizar una calificación jurídica existente para reforzar su posición, se asiste a una estrategia de ampliación de las capacidades de acción gracias a los interlocutores públicos y a los recursos jurídicos vinculados con ciertas formas de los espacios protegidos; se trataría, entonces, de una acción de empoderamiento por medio del espacio. Cuando los habitantes se movilizan para que se aplique una calificación jurídica sobre un subconjunto espacial, podría considerarse que se trata de la voluntad de conceder a ciertos sub-espacios cualidades jurídicas capaces de reforzar sus reivindicaciones y sus posiciones; en cierta forma, un empoderamiento del espacio.

\section{La actualización local del derecho}

En muchas entrevistas los grupos movilizados enarbolan una postura de vigilancia sobre prácticas y usos o sobre la acción de los poderes públicos. La eficacia de esta vigilancia, es traer la legislación - o la interpretación que se tiene de esta - en la situación local, de denunciar actividades ilegales o de hacer presión para que su interpretación de la situación sea trasformada en calificación jurídica.

Pero fuera de esta función de auxiliar de los autoridades públicas, la vigilancia de los habitantes permite mostrar la intensidad de la movilización y también inscribir en el sentido literal (cuando los vecinos multiplican cartas, quejas y denuncias) la preocupación social en el campo de la administración pública o del derecho. Hay la voluntad de pedir que se actualice el derecho, que los derechos otorgados por los textos sean llevados a la realidad. Me parece que los procesos que presento en este capitulo pueden caracterizarse como modalidades de actualización local del derecho.

Utilizo aquí la noción de actualización en un sentido próximo a su acepción filosófica o psicológica, de acción de hacer pasar del estado virtual al estado real para caracterizar la forma en la cual las reglas de derechos, los procedimientos, pueden ser activados, importados en una situación cuando ciertos actores los hacen existir movilizando las simbólicamente o prácticamente. Para nosotros, este concepto permite distinguir, en el caso de las transacciones alrededor de la construcción de reglas locales ligadas a negociaciones para salir de conflictos los procesos de inscripción local de recursos globales, de los que constituyen stricto sensu un proceso de producción local del derecho. Veremos que hasta acuerdos locales que se presentan como una producción jurídica constituyen más bien procesos de actualización local o de localización del derecho.

negociación y ajustes, tal y como queda demostrado en los diferentes textos recogidos sobre este tema en Territoires d'action (Melé y Larrue 2008). 
En varias situaciones analizadas, existe un marco de regulación de las actividades conformado por la existencia de comisiones consultativas o de participación, de seguimiento, de procedimiento de protección inscritos en los planes, de diferentes procedimiento de consulta o de información del público, se presentan o son analizadas por los habitantes movilizados como un marco particular resultado en parte de la movilización, para la toma en cuenta de sus derechos.

En muchos temas ambientales, el marco jurídico se encuentra en evolución rápida, esta evolución va en el sentido de una mejor protección del ambiente, de la implementación de medidas de control y de normas más precisas, así como de una mayor participación de la población. El objetivo de ciertas movilizaciones puede ser la implementación en una situación local de estas evoluciones. La evolución nacional del derecho constituye nuevos recursos de acción y viene legitimar sus reivindicaciones. La densidad de la movilización, y la calidad de la vigilancia es, para los habitantes, la condición para la aplicación rápida de nuevas normas nacionales.

Ciertas movilizaciones pueden centrar sus reivindicaciones - y presentar como un éxito local - una primera aplicación concreta de nuevos textos que no son todavía transformados en políticas públicas. Las controversias locales pueden basarse en la necesidad de encontrar un contenido, una modalidad de existencia local para elementos declarativos presentes en la ley. Estos elementos, pueden ser sin efectos reglamentarios, pero pueden constituir justificaciones de acción, recursos para reforzar una posición o una argumentación.

Podemos encontrar en nuestras entrevistas una tendencia a atribuir la implementación de procedimientos que son de hecho nacionales $-y$ a veces de carácter obligatorio - a la buena voluntad de los actores locales. Esta sobre valoración del papel de lo local pone a la luz la idea que la buena adaptación local de medidas reglamentarias nacionales exige una adhesión de las partes, una negociación entre los interés locales en particular cuando se trata de une mejor toma en cuenta de las reivindicaciones de los vecinos o habitantes. La idea se impone que el derecho no se aplica solo, que necesita ciudadanos que luchen por sus derechos.

\section{La productividad política de los conflictos urbanos}

\section{El conflicto produce actores políticos}

A este nivel, podemos identificar en nuestros estudios un primer efecto que podríamos denominar de politización. La participación de individuos a movilizaciones locales les confronta al mundo de la acción pública, entran en contacto con militantes, negocian con actores políticos, su experiencia del derecho cambia. Encontramos en las entrevistas, historias de politización en las cuales el involucramiento en un conflicto local se vuelve una modalidad de involucramiento en otras causas, de participación en otros grupos, de construcción de redes que pueden ser activadas en acceso a cargos políticos locales. Obviamente este tipo de trayectoria no es la más 
común en términos estadísticos, muchos no participan en conflictos, muchos participan o apoyan conflictos sin volverse militante o activistas. Una vez pasada la época de fuerte movilización con objetivos precisos, es posible presenciar un desencanto o solamente el repliegue de personas que no son dispuestos a pagar los importantes costos personales de una movilización. Sin embargo, la experiencia de la participación a una movilización constituye una experiencia de acción política que muchas veces se traduce par la participación a acciones o discusión en público sobre la definición del interés publico o del bien común.

Existen investigaciones interesantes sobre el papel de la socialización política de las movilizaciones, en los barrios, que subrayan que aún si no logran transformaciones sociales importantes, tienen la capacidad de modificar las relaciones de la población a la política. Sin embargo, hay que subrayar que el conflicto construye también un actor político colectivo, más o menos conectados con otros grupos (ONG, sindicatos, partidos, otras asociaciones de vecinos...). Según el principio de identidad definido por Alain Touraine (1973: 363) es posible destacar "que es el conflicto que constituye y organiza el actor" en el sentido que el conflicto constituye un actor colectivo consciente de lo que está en juego en las movilizaciones. Esta posición es muy próxima a lo que Alberto Melucci analiza como "identidad colectiva" (1999), es decir la capacidad colectiva - que se construye en el marco de interacción - de definirse a sí mismo de evaluar las oportunidades y los límites de la acción.

Como lo muestra Camille Hamidi (2006) es posible proponer una definición interaccionista de la politización por el cual es el proceso mismo de la acción en común, la definición del grupo pero también - para retomar el vocabulario de Nina Eliasoph et Paul Lichterman (2003) - , el estilo de los grupos, que permiten a sus miembros adoptar una definición compartida de la acción realizada en común. A esto se agrega lo que se espera de los participantes, de sus objetivos y de su relación con el exterior que constituye una forma de politización para los individuos que participan.

\section{Momentos de conflictos, de negociaciones, de participación}

Otra forma de pensar la dimensión política de los conflictos urbanos es de confrontar estas situaciones con los debates sobre la democracia participativa. Hasta este momento esta texto se ha referido a conflictos o movimientos urbanos pero en muchos contextos utilizamos este término para caracterizar situaciones que por momento son muy tensas y muy conflictuales, y por otros momentos son marcados por intercambios, negociación o por el involucramiento de los grupos movilizados en instancia de participación o de concertación.

Estos momentos del conflicto, de la negociación o de la participación están en muchos casos entrelazados. En Europa, América Latina o Estados Unidos, las situaciones de conflictos están asociadas a tentativas institucionales para reducirlas. Se tiene entonces que pensar una continuidad entre momentos de conflicto y momentos de participación. Me 
parece que tenemos que pensar en una forma de continuum entre conflicto y participación y se multiplican situaciones en las cuales la acción pública está en debate al interior y al exterior de las instancias de participación. Creadas para tratar los conflictos o construidas para ser espacios permanentes de participación estas instancias producen escenas sobre las cuales son puesta en debate las definiciones de un bien común territorializado; son también espacios de definición de las situaciones de negociación o de definición de lo aceptable para los diferentes actores.

Como lo propone J. Ion (2001: 17), estas situaciones se podrían llamar situaciones de acción pública, con una visión más extensiva de la noción de acción pública que no solo caracteriza la acción de los poderes públicos, pero el conjunto de las situaciones en las cuales la acción colectiva está sometida a debate público.

A este nivel podemos identificar un campo de reflexión muy interesante acerca de las relaciones entre conflictos y participación que permite resaltar algunas tensiones que producen los conflictos sobre los procedimientos de participación y algunos dilemas de la participación.

- El conflicto llena o vacía los espacios de participación : el conflicto permite llenar los espacios de participación con participantes interesados y evitar los procesos de participación vacíos que no encuentran su público; aunque en algunos casos el conflicto vacía los espacios de participación, ciertos grupos movilizados tienen una estrategia de no aceptar el debate, y de impedir la realización de debates considerados como simple formas de construcción de la aceptabilidad social.

- Los dispositivos de participación reducen y/o refuerzan los conflictos. Aun si las innovaciones institucionales han sido implementadas muchas veces para reducir los conflictos. Obviamente los procesos de participación, concertación no han permitido disminuir los conflictos. A pesar de innovaciones institucionales no existen contextos nacionales en los cuales se pude decir que la participación haya reemplazado el conflicto. Por otra parte se observa que los conflictos pueden encontrar en dispositivos de participación una escena para hacer valer su posición. En ciertos casos desde los poderes publicas se busca no desarrollar espacios de participación puesto que se tiene el temor de intensificar la conflictividad.

Estos elementos conducen también a intensos debates que cuestionan el papel de la participación, presentada a veces como un conjunto de dispositivos que buscan la despolitización o como dispositivos con capacidad de emancipación, obviamente no hay respuestas sino contextuales a esta disyuntiva (Gourgues, Rui, et Topçu 2013; Neveu 2011; Dagnino, Tatagiba 2010). 


\section{Conflictos y construcción de lo público}

Otro nivel de reflexión sobre la productividad política de los conflictos fue de considerar que los conflictos urbanos producen por su existencia misma un publicó - en el sentido de John Dewey (2004 [1927]) de grupo afectado que se vuelve activo - y por otra parte, una escena que contribuye a la emergencia y a la puesta a debate de un problema que por esta operación se vuelve un problema público.

Este nivel de problematización nos obliga a trabajar sobre las diferentes escenas sobre los cuales los actores llevan sus problemas, las cuestiones se encuentran a la base de la movilización. La capacidad de cada conflicto de construir un espacio público de debate depende de su capacidad a tener, a acceder a redes militantes o especializadas. Podemos considerar que es el conjunto de las acciones llevadas a cabo en un conflicto que producen a la vez el grupo movilizado, el público interesado y el espacio de debate en el cual un problema existe. Una gran parte de las acciones de los grupos movilizados se plantea mantener activo el debate, en el que podemos llamar siguiendo a Alberto Melucci (1999) un espacio público (político) intermediario. Podemos entonces considerar que los conflictos urbanos producen este espacio (político) público intermediario para poner en debate el porvenir de ciertos espacios.

No se trata entonces de invocar la idea de una opinión pública abstracta, pero de subrayar el papel de la construcción de un público organizado que constituye la escena pública sobre la base de diferentes formas de agrupación. Presentar las cosas así, permite intentar conceptualizar el papel del conflicto en la producción de las cuestiones públicas y de lo público sin encerrarse en el análisis del grado de apertura de los espacios públicos mediáticos o de los regímenes políticos.

Me parece también que permite rebasar el enfoque de la explicación de la incidencia de los conflictos a partir de un análisis de geografía política local (Subra 2007) o de ciencias políticas a partir de la noción de estructura de las oportunidades políticas, aun si obviamente es un nivel de reflexión interesante que en muchos casos tiene elementos de validez. A este nivel me parece importante destacar una analogía con lo que Jacques Rancière, filosofo francés, ha escrito en relación al movimiento obrero y de la fuerza de las reivindicaciones de reconocimiento de derechos y de igualdad que ha permitido el advenimiento del espacio de debate en el cual estas reivindicaciones son tomadas en cuenta. El sujeto obrero que evoca Rancière "para hacerse tomar en cuenta como interlocutor debe hacer como si la escena existe", "tiene que producir al mismo tiempo la argumentación y la escena en la cual tiene que ser escuchado, el objeto de la discusión y el mundo en el cual figura como objeto" (Rancière, 1995 : 55).

En los casos analizados, es el conjunto de las acciones de los grupos movilizados - manifestación, quejas, participación a reuniones públicas por parte de colectivos movilizados, escenas de negociación, de concertación o de participación - que constituyen a la vez el grupo movilizado, el público y el espacio de debate. 
En muchas de las situaciones estudiadas los grupos entrevistados subrayan el trabajo realizado para mantener activo el debate en el tiempo. La cuestión de la escala y de las formas de territorialización de este espacio público son, en varios de los casos determinante, algunos grupos pueden lograr que una cuestión rebase la escala local, para llegar a la capital del estado o regional, o ser debatido a nivel nacional o internacional.

Para los grupos movilizados el acceso al espacio público tiene efectos o implica ciertas necesidades. Para volver pública una cuestión, para transformarla en un problema público se necesita movilizar un público, hacer la demonstración que existe un público movilizado, adoptar una definición del interés público o de los "bienes comunes" y de movilizar la atención y la acción de los poderes públicos. Lo que implica un proceso de traducción de la cuestión en el lenguaje de los poderes públicos (Cefaï, 2002), en el vocabulario de las políticas públicas o del derecho, pero también de la necesidad de la legitimación de su tratamiento por los poderes públicos (Sheppard, 2004). Sin embargo no se debe concluir que cada situación de conflicto desemboca en la construcción de nuevos problemas públicos; ciertas movilizaciones pueden tomar la forma de la localización de problemas públicos o de causas que ya existen a nivel nacional o internacional (Chateauraynaud 2011).

\section{Conclusiones: Conflictos urbanos como experiencias de ciudadanía}

A manera de conclusión, quisiera evocar otra hipótesis de interpretación de la productividad de los conflictos urbanos: la participación en conflictos urbanos puede ser considerada como un acto (Isin, Nielsen, 2008) o una experiencia de ciudadanía ${ }^{9}$. Lo que nos lleva a conectar la cuestión de los efectos territoriales, jurídicos y políticos de los conflictos con el campo muy interesante de los nuevos estudios sobre la ciudadanía, que buscan rebasar el simple sentido institucional de la palabra para abarcar el sentido más extenso de la reivindicación del "derecho à tener derechos". Estos nuevos enfoques críticos (Neveu 2005, Clarke et all, 2014) plantean observar la construcción de la ciudadanía, fuera de los dispositivos institucionales, a partir de prácticas individuales o colectivas que cuestionan lo político y participan a la construcción de bienes comunes (Carrel, Neveu et lon, 2009). Las personas involucradas en conflictos expresan así directamente la manera en la cual piensan la ciudadanía y reivindican un papel de ciudadano (Carrel, 2007: p. 54).

En los estudios de casos, los actores utilizan muchas veces el término ciudadano para definir su posición de vigilancia y apoyar la legitimidad de su

\footnotetext{
${ }^{9}$ Referencia a la red de investigacion impulsada por Catherine Neveu "Expériences de citoyenneté ,entre ordinaire, politiques publiques et mobilisations"
} 
movilización. En un caso en Francia, una habitante declara para explicar la falta de involucramiento de vecinos frente a una decisión de extensión de un relleno sanitario declara : "en esta época, el ciudadano no hizo su trabajo" (Cirelli, 2013). Los grupos movilizados muchas veces reivindican ejercer una cualidad particular de la ciudadanía más completa que la de aquellos habitantes que simplemente votan, una ciudadanía preocupada del porvenir del espacio del barrio o de los espacios públicos como bien común. JeanMichel Fourniau (2007) a partir de trabajos sobre controversias acerca de proyectos de infraestructura, subraya la importancia, en los argumentos presentados por las asociaciones locales, del agravio a la calidad de ciudadano toda vez que un proyecto no tome en cuenta sus usos, intereses y opiniones. Propone considerar la existencia de una nueva figura de ciudadano que se define a partir a su calidad de "vecino" y que reivindica una participación a las decisiones públicas. Esta ciudadanía activa que se expresa como la voluntad de participar en la definición de un interés público local (Jobert , 1998) tiene a la vez dimensiones de territorialidad, de relación de apropiación y de identificación con un espacio y de relación a lo público .

En el contexto de redefinición del papel del Estado en la América Latina de los años 1980 y 1990, los movimientos urbanos reivindican una concepción amplia de la ciudadanía que rebasa el simple derecho de voto y incluya derechos de acceso a las infraestructuras y los servicios urbanos, como así derechos complejos como un "derecho a la ciudad" que se puede definir en este caso como un derecho a participar en los debates acerca del futuro de los espacios urbanos. Ciertos autores analizan la evolución de los objetivos de los conflictos urbanos como la transición de luchas directamente asociadas al acceso al suelo, a la vivienda o a los servicios urbanos a conflictos más bien vinculado a las modalidades de regulación de la ciudad (Renna Gallano 2008). Las movilizaciones urbanas pueden entonces ser vistas como la constitución de nuevas formas de ciudadanía, definida como la capacidad a constituirse en sujeto político (Dagnino 2004), es decir a salir de la exclusión y de la sub-integración urbana. Los conflictos urbanos pueden ser analizados como momentos de acción colectiva que se traducen por un empoderamiento de los grupos movilizados y por una más grande capacidad de acción. Sin embargo, se debe apuntar que la capacidad de movilización puede ser muy diferente de un contexto al otro como los efectos sobre los grupos que intentan organizar. En numerosos casos, se debe relativizar el relato del acceso a la ciudadanía y de una conciencia participativa vinculada a las movilizaciones como lo subrayan Daniel Levine et Catalina Romero (2004) que momentos de des-empoderamiento pueden suplantar momentos de movilización. Los lideres pueden agotar sus fuerzas o seguir otro camino, y los habitantes movilizados necesitar retomar una vida centrada sobre el grupo familiar.

Sin embargo, si aún el debate queda abierto sobre lo que producen los conflictos analizados a largo plazo y si las consideraciones descontextualizadas que presente aquí tendrían que ser matizadas y ancladas en los contextos nacionales y locales, intenté mostrar que las situaciones de conflicto constituyen hoy en día un objeto privilegiado para la 
investigación urbana puesto que permiten, por una parte, observar la forma en la cual grupos de habitantes o de militantes intentan construirse como actores $\mathrm{y}$, por otra parte, contribuir a un mejor entendimiento de las dinámicas urbanas tomando en cuenta las controversias sobre el porvenir de los espacios urbanos.

\section{Bibliografía}

AMIOT Michel, 1986. Contre l'Etat les sociologues, Eléments pour une histoire de la sociologie urbaine en France (1900-1980). Paris: éditions de l'École des Hautes Études en Sciences Sociales.

AZUELA Antonio, MELÉ Patrice, UGALDE Vicente, 2015. "Conflits de proximité et rapport(s) au(x) droit(s)". Développement durable et territoires. Économie, géographie, politique, droit, sociologie. 2015. №1.

BAREL Yves, 1984. La société du vide. Paris: Seuil.

BASSOLS Mario, MELÉ Patrice, 2001. Medio ambiente, ciudad y orden jurídico. México: UAM, Miguel Angel Porrua.

BECK Ulrich, 2006. La sociedad del riesgo global, Madrid, Siglo XXI, 300 p. (primera edición 1986).

BOBBIO Luigi, 2011. "Conflitti territoriali: sei interpretazioni ». Tema. Journal of Land Use, Mobility and Environment, 4.

BOURDIN Alain, LEFEUVRE Marie-Pierre, MELÉ Patrice 2006. L'élaboration des règles du jeu urbain. en, Les règles du jeu urbain, entre droit et confiance. Paris: Descartes et cie. p. 21-49.

CARREL Marion, 2007. La citoyenneté urbaine du point de vue des gouvernés, Paris: Institut Marcel Mauss (CEMS-EHESS), Atelier PUCACNRS citoyenneté urbaine.

CARREL Marion, NEVEU Catherine, ION Jacques. dir., 2009. Les intermittences de la démocratie. Formes d'action et visibilités citoyennes dans la ville, Paris: L'Harmattan.

CASTELLS Manuel, 1974. Movimientos sociales urbanos. Madrid: Siglo Veintiuno de España.

CASTELLS Manuel, 1983. The city and the grassroots : a cross-cultural theory of urban social movements. London: E. Arnold, Berkeley, University of California Press.

CEFAÏ Daniel, 2002. "Qu'est-ce qu'une arène publique ? Quelques pistes pour une approche pragmatique », en, D. Cefaï et I. Joseph, L'héritage du pragmatisme. Conflits d'urbanité et épreuves de civisme, La Tour d'Aigues, Éditions de l'aube, p. 51-79.

CIRELLI Claudia, 2013. "Elle était petite, elle ne se voyait pas" : mobilisation et conflit autour d'une décharge en Isère », en, P. MELÉ (éd.), 2013. Conflits de proximité et dynamiques urbaines. Rennes, PUR, p. 95135. 
CLARKE John, COLL Kathleen, DAGNINO Evelina, NEVEU Catherine, 2014. Disputing citizenship, Bristol: Policy Press.

CHATEAURAYNAUD Francis, 2011. Argumenter dans un champ de forces, essai de balistique sociologique. Petra.

COSER Lewis A., 1982. Les fonctions du conflit social, Paris: Puf. (primera edicion en inglés 1956).

DAGNINO E., 2004. "¿ Sociedade civil, participação e cidadania: de que estamos falando ?", en, Políticas de ciudadanía y sociedad civil en tiempos de globalización, Caracas: Daniel Mato, p. 95-110.

DAGNINO E., TATAGIBA L., 2010. "Mouvements sociaux et participation institutionnelle: répertoires d'action collective et dynamiques culturelles dans la difficile construction de la démocratie brésilienne". Revue internationale de politique comparée, 2010, vol. 17, n³, p. 167-185.

DEAR Michael, 1992. "Understanding and Overcoming the NIMBY Syndrome". Journal of the American Planning Association. Vol. 58, $\mathrm{n}^{\circ} 3$, p. 288-300.

DEWEY John, 2004. La opinión pública y sus problemas. Madrid: Ediciones Mortara, (primera edición en inglés 1927).

DUHAU Emilio, 2012. "Les conflits de proximité au Mexique: entre mobilisation de rue et mobilisation du droit", Géocarrefour. Vol. 87, n 1.

DUHAU Emilio, GIGLIA Angela, 2008. Las reglas del desorden: habitar la metrópoli: la ciudad de México a principio del siglo XXI. México: Siglo XXI, UAM Azcapotzalco.

ELIASOPH Nina, LICHTERMAN Paul, 2003. "Culture in Interaction". American Journal of Sociology. enero. Vol. 108, n 4, p. 735-794.

FILLIEULE Olivier, MATHIEU Lilian, PECHU Cécile, 2009. Structure des opportunités politiques. Paris: Presses de sciences po.

FOURNIAU, Jean-Michel, 2007. "L'expérience démocratique des « citoyens en tant que riverains » dans les conflits d'aménagement". Revue européenne des sciences sociales. Vol. XLV, p. 149-179.

GIDDENS Anthony, 1995, La constitución de la sociedad, Madrid, Amorrortu Editores, $416 \mathrm{p}$ (primera edición en inglés, 1984) .

GIDDENS Anthony, 2008. Consecuencias de la modernidad, Madrid, Alianza Editorial, (primera edición en inglés, 1994).

GOIRAND Camille, 2010. "Penser les mouvements sociaux d'Amérique latine", Revue française de science politique, vol. 60, n³, p. 445-466.

GOURGUES Guillaume, RUI Sandrine, TOPÇU Sezin, 2013. "Gouvernementalité et participation », Participations, 2013, vol. N 6, n², p. 5-33. 
HAMIDI Camille, 2006. «Éléments pour une approche interactionniste de la politisation ». Revue française de science politique. Vol. 56, n¹, p. 5-25.

HARVEY David, 2013. Ciudades rebeldes del derecho de la ciudad a la revolución urbana, Madrid, Akcal, 237 p.

ION Jacques, 2001. "Introduction », en, J. Ion (dir.), L'engagement au pluriel, Saint-Etienne, Presse de I'Université de Saint-Etienne, p.10-20.

ISIN Engin F., NIELSEN Greg M. (dir.) 2008. Acts of Citizenship, Londres: ZedBooks.

JOBERT, Arthur, 1998. "L'aménagement en politique. Ou ce que le syndrome NIMBY nous dit de l'intérêt général". Politix Vol. 11, p. 67-91.

LE BERRE Maryvonne, 1992. "Territoires », en A. Bailly, R. Ferras, D. Pumain, Encyclopédie de la géographie, Paris, Economica, p. 617-633.

LEVINE Daniel, ROMERO Catalina., 2004. "Movimientos urbanos y desempoderamiento en Perú y Venezuela", América Latina Hoy, $n^{\circ} 036$, p. 47-77.

LOLIVE Jacques, 1999. Les contestations du TGV méditerranée, Paris: L'Harmattan.

LYMAN Stanford M., Marvin B. Scott, " Territoriality : a neglected sociological dimension ", Social problems, vol. 15, n², 1967, p. 236-249.

MELÉ Patrice, 2001. "Monterrey: medio ambiente y urbanización en una metrópoli industrial", en, Mario Bassols, Patrice Melé (Coord.), Medio ambiente, ciudad y orden jurídico, UAM: Miguel Angel Porrua, Mexico, p. 51118.

MELÉ Patrice (éd.), 2013. Conflits de proximité et dynamiques urbaines. Rennes: PUR.

MELÉ Patrice, 2009. "Identifier un régime de territorialité réflexive », en, Martin Vanier (coord.), Territoires, territorialité, territorialisation ; controverses et perspectives, Rennes, PUR, 2009, p. 45-55.

MELÉ Patrice, 2006. « Lutter contre les bruits de la ville, mobilisation du droit et production d'ordres locaux », en, A. Bourdin, M-P. Lefeuvre, Les règles du jeu urbain, entre droit et confiance. Paris: Descartes et cie, p. 207-242.

MELÉ Patrice, 2010. "Las dimensiones conflictivas del patrimonio », en, Gestionar el patrimonio en tiempo de la globalización. México : Biblioteca de Alteridades, UAM Iztapalapa, Juan Pablos editores. p. 123-160.

MELÉ Patrice, LARRUE Corinne, ROSEMBERG Muriel, 2003. Conflits et territoires. Tours: PUFR.

MELÉ Patrice, LARRUE Corinne (coord.) 2008, Territoires d'action. Paris: L'Harmattan.

MELÉ, Patrice, 2012. "Pour une géographie des conflits urbains de proximité en Amérique Latine». Géocarrefour. Vol. Vol. 87, n 1, p. 3-13. 
MELÉ, Patrice, 2014. Transacciones territoriales, Patrimonio, medio ambiente y acción pública en México. México : UAM Iztapalapa, CEMCA, Juan Pablo Editor.

MELÉ Patrice, AZUELA Antonio, GERMAIN Annick et all, 2013. « Analyser la productivité des conflits de proximité, en, P. MELÉ (éd.), 2013. Conflits de proximité et dynamiques urbaines. Rennes, PUR, p. 389-429.

MELUCCI Alberto, 1999. Acción colectiva, vida cotidiana y democracia, México, El Colegio de México, 1999.

MESSU, Michel, 2012. "Explication sociologique et domination sociale ». SociologieS [en ligne]. noviembre.

MOCTEZUMA Pedro, 1994. "El movimiento urbano popular mexicano, Nueva Anthropologia, vol. VI, n²4, p. 61-85.

MOCTEZUMA Pedro, 1999. Despertares, comunidad y organización urbano popular en México, 1970-1994. México: Universidad Iberoamericana, UAM.

MOYA Carlos, 1982. Teoría sociológica : una introducción critica, Madrid: Taurus.

NEVEU Catherine, 2011. " Démocratie participative et mouvements sociaux : entre domestication et ensauvagement? ", Participations, 2011, vol. 1, p. 188-211.

NEVEU Catherine, 2005. Anthropologie de la citoyenneté, Université de Provence, Aix-Marseille.

RANCIĖRE Jacques, 1995. La Mésentente. Politique et philosophie, Paris: Galilée.

RUI Sandrine, 2013. "Où donc est le danger? », Participations, vol. N 6, n², p. 65-86.

RENNA GALLANO H., 2008. (Vi)viendo la Lucha por la Ciudad: Actores y Conflictos Urbanos en América Latina y en la Ciudad de Santiago Chile. Sur, Documento de Trabajo, s/p.

SACK Robert D., 1986. Human territoriality : its theory and history. NewYork: Cambridge University Press.

SHEPPARD Elisabeth, 2004. "Problèmes publics », en Boussaguet L., Jacquot S. et Ravinet P. (dir.), Dictionnaire des politiques publiques, Paris: Presses de Sciences Po, p.530-538

SIMMEL Georg, 2010. El conflicto. Sociología del antagonismo. Madrid: Sequitur (primera edición 1903).

SOJA Edward William, 2010. Seeking spatial justice. Minneapolis: University of Minnesota Press.

STANFORD M. Lyman, SCOTT Marvin B., « Territoriality : a neglected sociological dimension », Social problems, vol. $15, n^{\circ} 2,1967$, p. 236-249. 
SUBRA Philippe, 2007. Géopolitique de l'aménagement du territoire. Paris: Armand Colin.

THÉVENOT Laurent, 2006. L'action au pluriel, sociologie des régimes d'engagement. Paris: La découverte.

TILLY Charles, TARROW Sidney, 2006. Contentious Politics, Paradigm Publishers.

TOURAINE Alain, 1973. Production de la société. Paris: Fayard.

TROM Danny, 1999. « De la réfutation de l'effet NIMBY considérée comme une pratique militante. Notes pour une approche pragmatique de l'activité revendicative ", Revue française de science politique. 1999. Vol. 49, p. $31-50$.

VALLADARES L., PRATES CUELHO M., 1995, La investigación urbana en América Latina. Tendencias actuales y recomendaciones, Gestión de las transformaciones sociales MOST, Documentos de debates, $\mathrm{N}^{\circ} 4$, Paris, UNESCO.

WOLSINK, Maarten, 1994. "Entanglement of Interests and Motives: Assumptions behind the NIMBY-theory on Facility Siting ». Urban Studies. Vol. 31, $n^{\circ} 6$, p. 851-866. 